\title{
МОДЕЛЬ РАДИОЛИНИЙ С ИСПОЛЬЗОВАНИЕМ ПРОСТРАНСТВЕННО-ВРЕМЕННОЙ ОБРАБОТКИ ШИРОКОПОЛОСНЫХ СИГНАЛОВ ДЛЯ НУЖД ДОЛЖНОСТНЫХ ЛИЦ СИТУАЦИОННЫХ ЦЕНТРОВ КОМПЛЕКСНОЙ СИСТЕМЫ БЕЗОПАСНОСТИ
}

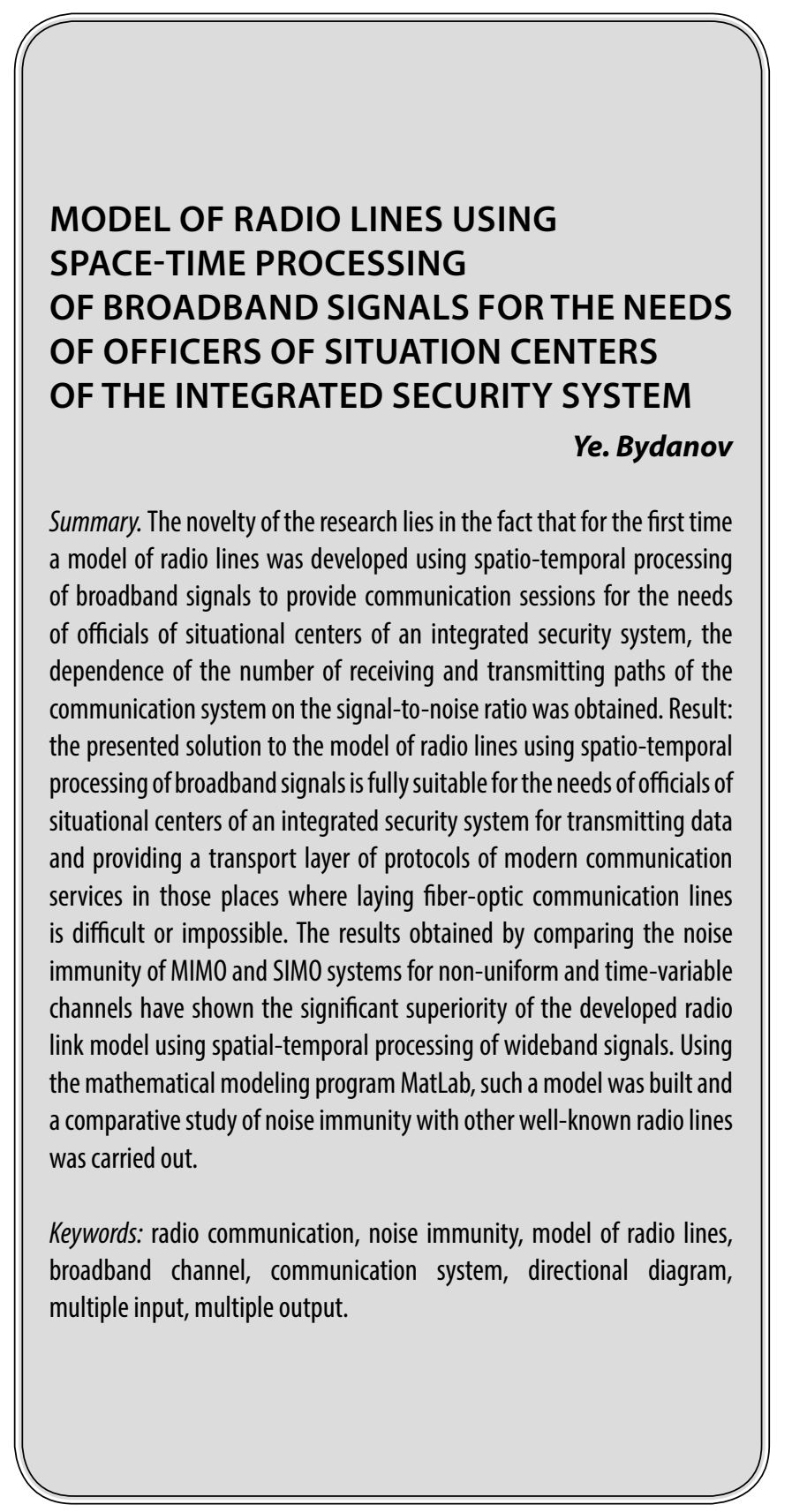

Быданов Егор Владимирович 1

Адъюнкт, ФГКВОУ ВО «Военная академия связи имени маршала Советского Союза С. М. Буденного» Министерства обороны Российской Федерации, Санкт-

Петербург

egorius.90@mail.ru

Аннотация. Новизна исследования заключается в том, что впервые разработана модель радиолиний с использованием пространственно-временной обработки широкополосных сигналов для предоставления сеансов связи для нужд должностных лиц ситуационных центров комплексной системы безопасности, получена зависимость числа приёмных и передающих трактов системы связи от величины соотношения сигнал/шум. Результат: представленное решение модели радиолиний с использованием пространственно-временной обработки широкополосных сигналов полностью подходит для нужд должностных лиц ситуационных центров комплексной системы безопасности для передачи данных и предоставления транспортного уровня протоколов современных сервисов связи в тех местах, где прокладка оптоволоконных линий связи затруднена или невозможна. Результаты, полученные путем сравнения помехоустойчивости систем MIMO и SIMO, для неоднородных и непостоянных во времени каналов, показали значительное превосходство разработанной модели радиолинии с использованием пространственно-временной обработкой широкополосных сигналов. С помощью программы математического моделирования MatLab такая модель была построена и проведено сравнительное исследование помехоустойчивости с другими известными радиолиниями.

Ключевые слова: радиосвязь, помехоустойчивость, модель радиолиний, широкополосный канал, система связи, диаграмма направленности, множественный вход, множественный выход. 


\section{Ввемение}

$\longrightarrow$ егодня от всех силовых структур требуется постоянная готовность к выполнению мер обеспечения безопасности государства. При этом большая часть задач возлагается на ситуационные центры различных ведомств. Для функционирования ситуационных центров требуется оборудование, построенное на основе современных технологий связи, способное передавать большие объемы информации с высоким качеством, в связи с чем предъявляются более высокие требования к каналам связи по пропускной способности и помехоустойчивости [3]. Отметим, что не все технологии и оборудование пригодны для использования, т.к. при выборе технических средств необходимо выполнить ряд требований по защите информации от утечек по каналам побочных электромагнитных излучений и наводок (ПЭМИН) [2], а также по защите должностного лица от электромагнитных излучений $[1,4]$.

Сегодня ситуационные центры оснащены оборудованием связи, работа которого основана на применении технологии SISO (Single Input Single Output) с одной антенной на передаче и приеме. Учеными ведущих стран мира велись исследования по разработке беспроводных систем, способных к высокой скорости передачи информации и возможности предоставления современных сервисов связи. Широко исследуются такие технологии, как MISO (Multiple Input Single Output) и SIMO (Soft Input Multiple Output). В работе [7, 9] описана передача информации по разнесенным в пространстве каналам системы MIMO (Multiple Input Multiple Output). В литературе $[6,8]$ широко исследована Шенноновская пропускная способность, однако нигде не представлено сравнение результатов помехоустойчивости системы связи MIMO с различным числом приемных и передающих трактов с радиолиниями другими технологий. Таким образом, возникает необходимость в разработке радиолинии, с помощью которой должностные лица смогли бы использовать современные сервисы связи для решения повседневных задач, тем более в тех местах, где не имеется возможность прокладки проводных и оптоволоконных линии связи, при этом скорость передачи информации и помехоустойчивость (коэффициент битовой ошибки составляет $10^{9}$ ) немного уступали бы им [5]. Цель работы - создание модели радиолиний с использованием пространственно-временной обработки широкополосных сигналов, при помощи которой возможно устанавливать сеансы связи между должностными лицами ситуационных центров комплексной системы безопасности с поддержкой современных сервисов связи. Расчеты экспериментальных исследований на основе компьютерного моделирования проводятся для оценки помехоустойчивости созданной модели в сравнении с известными радиолиниями ситуационных центров, функционирование которых основано на базе технологии SISO.

\section{Постановка заАачи}

Пусть спектральная плотность шумов в пределах полосы пропускания на частоте $n$ радиоприёмных трактов сохраняет постоянное значение. Пусть есть $v$ выборки сигнала $S_{I}(k) \ldots \mathrm{S}_{v}(k)$, принятые в $n$ независимых каналах приёма (полученные от $m$ передающих трактов), содержащие по $K$ комплексных отсчетов на частоте дискретизации $F_{s}$ :

$$
\begin{aligned}
& S_{1}(k)=s_{1}(k)+q_{1}(k), \\
& S_{2}(k)=s_{2}(k)+q_{2}(k)=\alpha_{2} \cdot s_{1}\left(k+D_{1,2}\right)+q_{2}(k), \\
& S_{v}(k)=s_{v}(k)+q_{v}(k)=\alpha_{v} \cdot s_{1}\left(k+D_{1, v}\right)+q_{v}(k),
\end{aligned}
$$

где $s_{l}(k)$ - комплексная огибающая сигнала моделируемой радиолинии системы, принятого первым трактом приёма; $s_{2}(k)$ - комплексная огибающая сигнала моделируемой радиолинии системы, принятого вторым трактом приёма с коэффициентом затухания $\alpha_{2} ; s_{v}(k)$ - комплексная огибающая сигнала моделируемой радиолинии системы, принятого $v$-трактом приёма с коэффициентом затухания $\alpha_{v} ; q_{1}(k), q_{2}(k)$ - аддитивные гауссовские центрированные шумы в первом, втором и $v$-тракте соответственно; $D_{1,2}, D_{l, v}$ задержка сигнала $s_{l}(k)$ относительно $s_{2}(k)$ и сигнала $s_{l}(k)$ относительно $s_{v}(k)$ выраженная в отсчетах сигнала. Отметим, что сигнал может быть некоррелированный с шумами $q_{1}(k), q_{2}(k) \ldots q_{v}(k)$.

Входными данными модели радиолинии являются:

- $m$ - число передающих трактов системы связи построенной на основе технологии MIMO;

- $n$ - число приёмных трактов системы построенной на основе технологии МІMO;

- $M$ - номер, соответствующий виду модуляции сигнала ортогональных подканалов, которые передаются параллельно;

- $P_{\text {мин }} . P_{\text {макс }}$ - диапазон соотношения $E_{b} / N_{0}$, выраженное в децибелах для моделирования сигнала;

- $T_{\text {cu }}$ - длительность генерируемого сигнала $s(k)$ для расчёта коэффициента битовой ошибки;

- $R_{\text {инф }}$ - информационный поток размером $L_{u н \phi}$ бит, который подается на модулятор, в зависимости заданного вида модуляции М формируется сигнал $s(k)$;

- $R_{c u x}$ - на приемной стороне именно по этому сигналу осуществляется корреляция сигнала для различных временных и частотных сдвигов, до тех пор пока не появится пик в огибающей взаимной корреляционной функции (ВКФ) [10, 11]. 


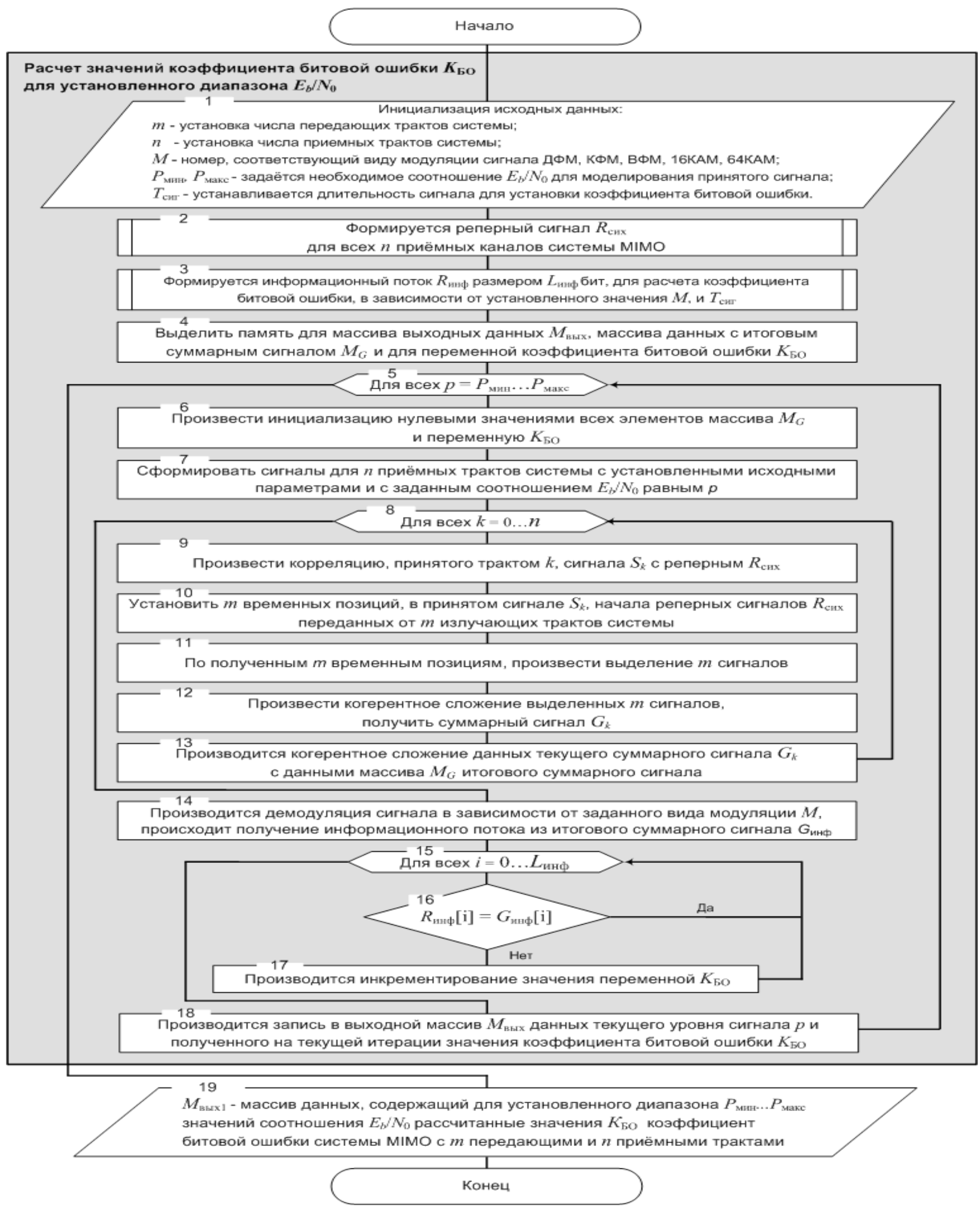

Рис. 1. Схема алгоритма расчета помехоустойчивости для системы MIMO с n приемными и передающими трактами 


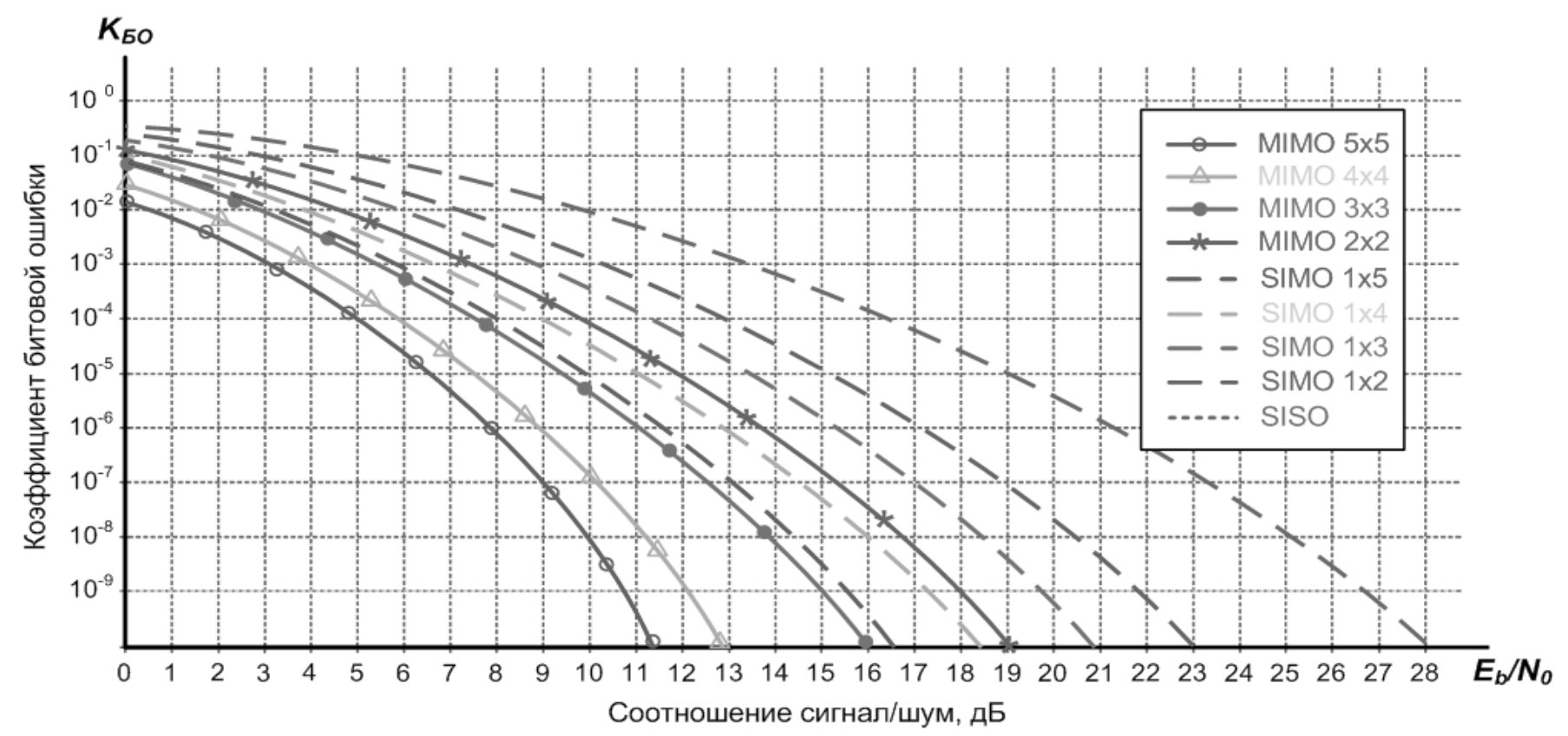

Рис. 2. Зависимость значений коэффициента битовой ошибки КБО от величины Eb/N0 для систем MIMO, SIMO, SISO, модуляция ДФМ

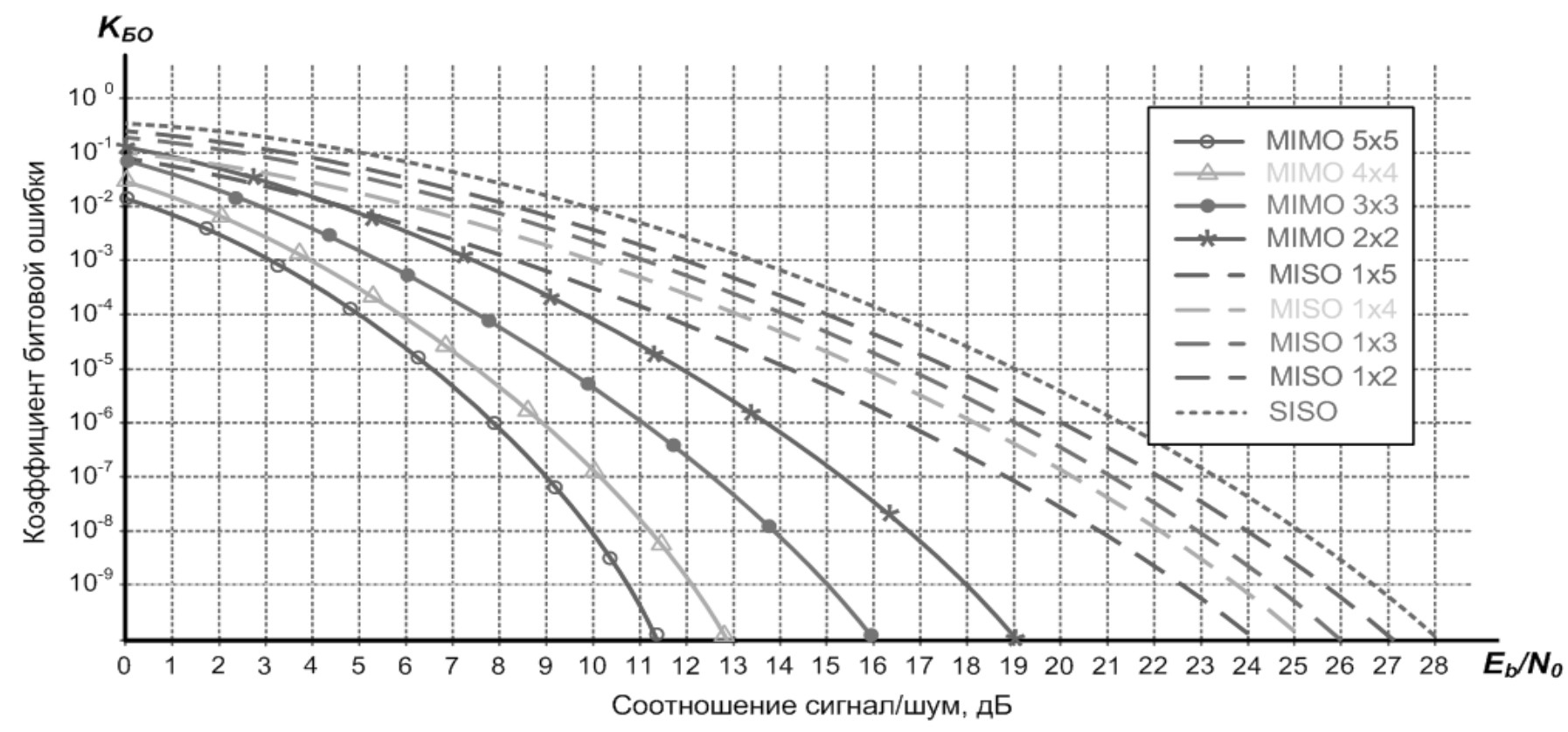

Рис. 3. Зависимость значений коэффициента битовой ошибки КБО от величины Eb/N0 для систем MIMO, MISO, SISO, модуляция ДФМ

Решение. Схема алгоритма сравнения помехоустойчивости систем MIMO и SIMO представлена на рис. 1. Рассмотрим его основные блоки. Сначала инициализируются исходные данные, необходимые для формирования массива выходных данных дискретной модели, блок 1 на рис. 1. В блоке 2 на рис. 1 происходит формирование реперного сигнала $R_{\text {cux }}$ В блоке 3 на рис. 1 формируется информационный поток $R_{u н \phi}$ объемом $L_{u н \phi}$ бит в зависимости от установленного значения $M$ и $T_{c u г}$. Данный информационный поток и будет передаваться по каналам связи системы. В блоке 4 на рис. 1 происходит вы-

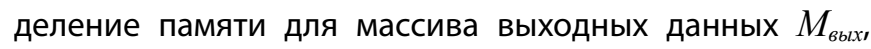


массива данных с итоговым суммарным сигналом $M_{G}$ и для переменной коэффициента битовой ошибки $K_{Б O}$. При помощи цикла 5 на рис. 1 осуществляется перебор, инкрементированием на единицу, диапазона $P_{\text {мин }} . . P_{\text {макс }}$ заданных уровней сигнала. В блоке 6 на рис. 1. происходит инициализация нулевым значением переменной $K_{Б о}$ и всех элементов массива $M_{G}$. В блоке 7 на рис. 1 происходит формирование $n$ сигналов для $n$ приёмных трактов системы с установленными исходными параметрами и с заданным соотношением $E_{b} / N_{0}$ равным $p$. При помощи цикла 8 на рис. 1 осуществляется выполнение одинаковых действий для всех $n$ приёмных трактов системы: в блоке 9 на рис. 1 выполняется корреляция, принятого трактом $k$, сигнала $S_{k}$ с реперным $R_{c u x}$ для определения $m$ временных позиций в принятом сигнале $S_{k}$ блок 10 на рис. 1 (таким образом, определяются на-

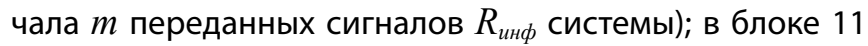
на рис. 1 происходит выделение всех найденных $m$ сигналов; в блоке 12 на рис. 1 производится когерентное сложение всех выделенных сигналов $m$ между собой, в результате сложения получается суммарный сигнал тракта $k$, который может быть, в свою очередь, когерентно сложен с сигналами других $n$ - 1 трактов и итоговый сигнал в блоке 13 на рис. 1 сохраняется в массиве данных $M_{G}$. В блоке 14 на рис. 1 производится демодуляция итогового сигнала из массива данных $M_{G}$ в зависимости от заданного вида модуляции $M$, полученный битовый поток информационных данных будем обозначать

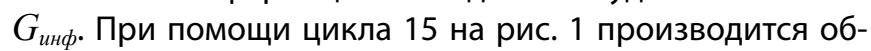
ход всех информационных бит потока $G_{u н \phi}$ и в блоке 16 на рис. 1 происходит сравнение их с заданным потоком $R_{\text {инф. }}$ В случае если информационные биты в одинаковых позициях $i$ не равны, то в блоке 17 на рис. 1 производится инкрементирование коэффициента битовой ошибки $K_{5 O}$. Таким образом, по завершению выполнения цикла 15 на рис. 1 и действий внутри него в блоке 18 на рис. 1 устанавливается, в выходной массив данных $\mathrm{M}_{\text {выx }}$, конечное значение коэффициента битовой ошибки $K_{5 O}$ системы MIMO с $m$ передающими и $n$ приёмными трактами. По завершению всех итераций цикла 8 на рис. 1 в блоке 19 на рис. 1 данные массива $M_{\text {выx }}$ готовы для последующего анализа.

На рис. 2-3 представлены графики, на которых наглядно отображены рассчитанные значения массива данных $\mathrm{M}_{\text {выхl }}$ по представленному выше алгоритму коэффициента битовой ошибки $K_{Б O}$. По представленным графикам появляется возможность наглядно оценить помехоустойчивость систем MIMO, SIMO, SISO, MISO.

На рис. 2 видно, что при $\mathrm{K}_{Б O}$, равному $10^{-5}$, выигрыш между MIMO 5x5 и SIMO 1×5 составляет 4 дБ; выигрыш между MIMO 4x4 и SIMO 1×4 составляет 3 дБ, выигрыш между MIMO 3×3 и SIMO 1×3 равен 4 дБ, выигрыш между MIMO 2×2 и SIMO 1×2 равен 6 дБ.
На рис. 3 видно, что при $\mathrm{K}_{Б о}$, равному $10^{-5}$ выигрыш между MIMO 5×5 и MISO 1×5 составляет 8 дБ; выигрыш между MIMO 4x4 и MISO 1x4 составляет 8 дБ, выигрыш между MIMO 3×3 и MISO 1×3 равен 7 дБ, выигрыш между MIMO 2x2 и MISO 1×2 равен 6 дБ.

Как показывает анализ представленных графиков на рис. 2-3, с увеличением числа приёмных трактов помехоустойчивость передачи данных увеличивается. Для того, чтобы определить число приёмных трактов, при котором соотношение $E_{b} / N_{0}$ будет минимальным для значения коэффициента битовой ошибки $K_{50}$, соответствующего оптоволоконной линии связи $10^{-9}$. Произведем расчеты по алгоритму, представленному на рис. 4 и по полученным данным построим график исследуемой зависимости для последующего анализа.

Рассмотрим основные блоки алгоритма, представленного на рис. 4. Сначала инициализируются исходные данные (блок 1 на рис. 4). В блоке 2 на рис. 4 происходит формирование реперного сигнала $R_{c u x}$, на приемной стороне именно по этому сигналу осуществляется корреляция сигнала для различных временных и частотных сдвигов до тех пор, пока не появится пик в огибающей взаимной корреляционной функции (ВКФ).

В блоке 3 на рис. 4 формируется информационный поток $R_{\text {инф }}$ размеров $L_{\text {инф }}$ бит в зависимости от установленного значения $M$ и $T_{c u r}$. Данный информационный поток и будет передаваться по каналам связи системы. В блоке 4 на рис. 4 происходит выделение памяти для массива

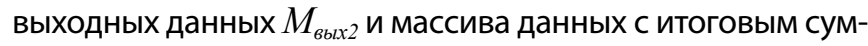
марным сигналом $M_{G}$ и для переменной коэффициента битовой ошибки $K_{5 O}$. При помощи цикла 5 на рис. 4 осуществляется перебор инкрементированием на единицу диапазона $1 \ldots N$ заданного числа передающих и приёмных трактов системы. Для каждого $n \in[1 ; N)$ устанавливается значение соотношения $E_{b} / N_{0}$, при котором коэффи-

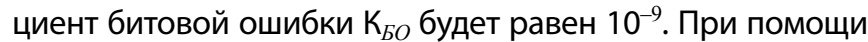
цикла 6 на рис. 4 осуществляется перебор инкрементиро-

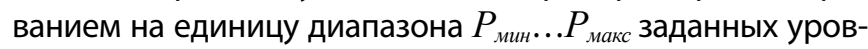
ней сигнала. В блоке 7 на рис. 4 происходит инициализация нулевым значением переменной $K_{5 O}$ и всех элементов массива $M_{G}$. В блоке 8 на рис. 4 происходит формирование $n$ сигналов для $n$ приёмных трактов системы с установленными исходными параметрами и с заданным соотношением $E_{b} / N_{0}$ равным $p$. При помощи цикла 9 на рис. 4 осуществляется выполнение одинаковых действий для всех $n$ приёмных трактов системы. При помощи цикла 9 на рис. 4 осуществляется выполнение одинаковых действий для всех $n$ приёмных трактов системы. Таким образом, определяются начала $n$ переданных сигналов $R_{\text {инф }}$ системы.

В блоке 12 на рис. 4 происходит выделение всех найденных $n$ сигналов; в блоке 13 на рис. 4 производится ко- 


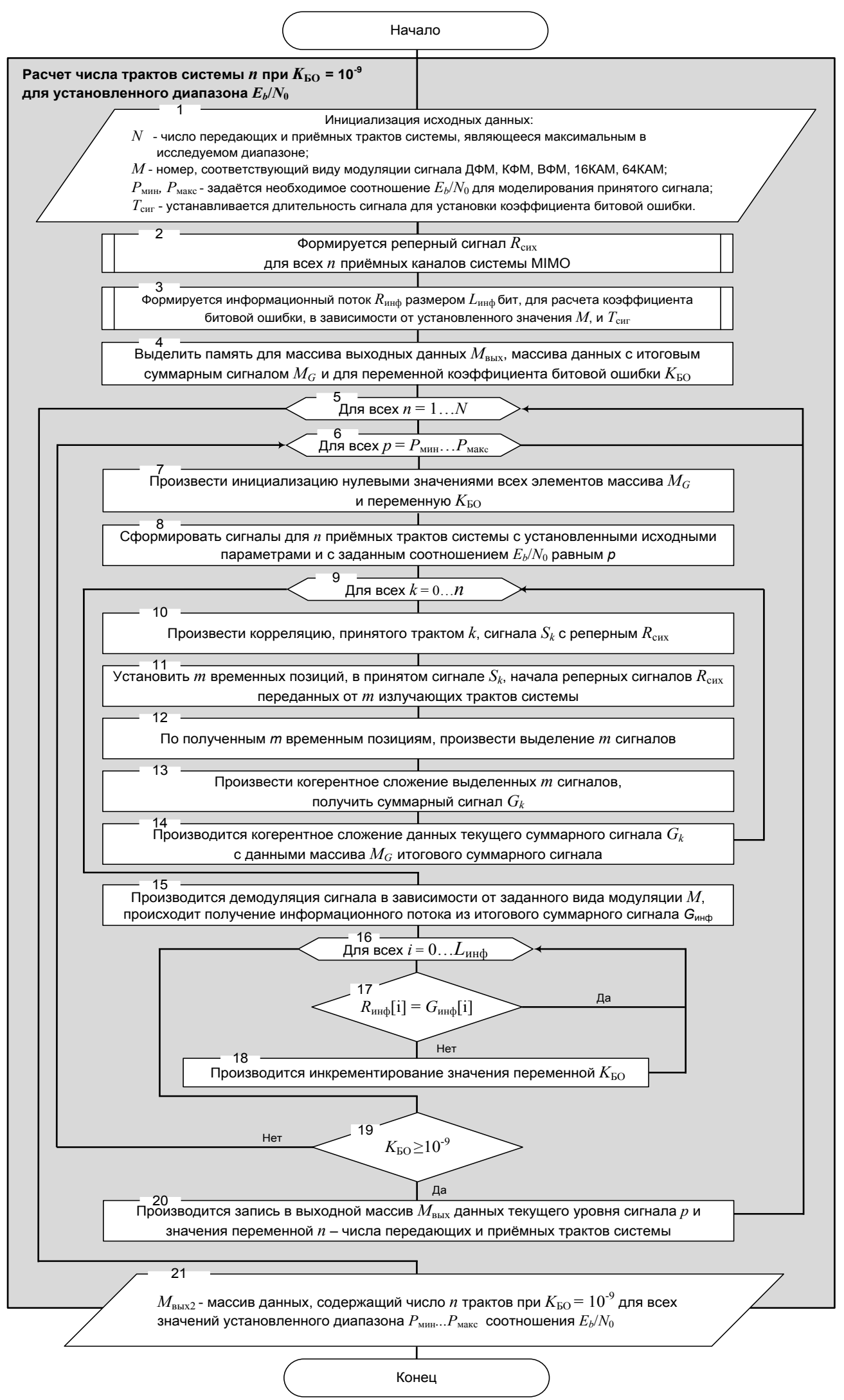

Рис. 4. Схема алгоритма расчета приемных трактов $n$ для системы MIMO, при котором соотношение $E_{b} / N_{0}$ будет минимальным для значения коэффициента битовой ошибки $K_{b O}$ соответствующего оптоволоконной линии связи $10^{-9}$ 


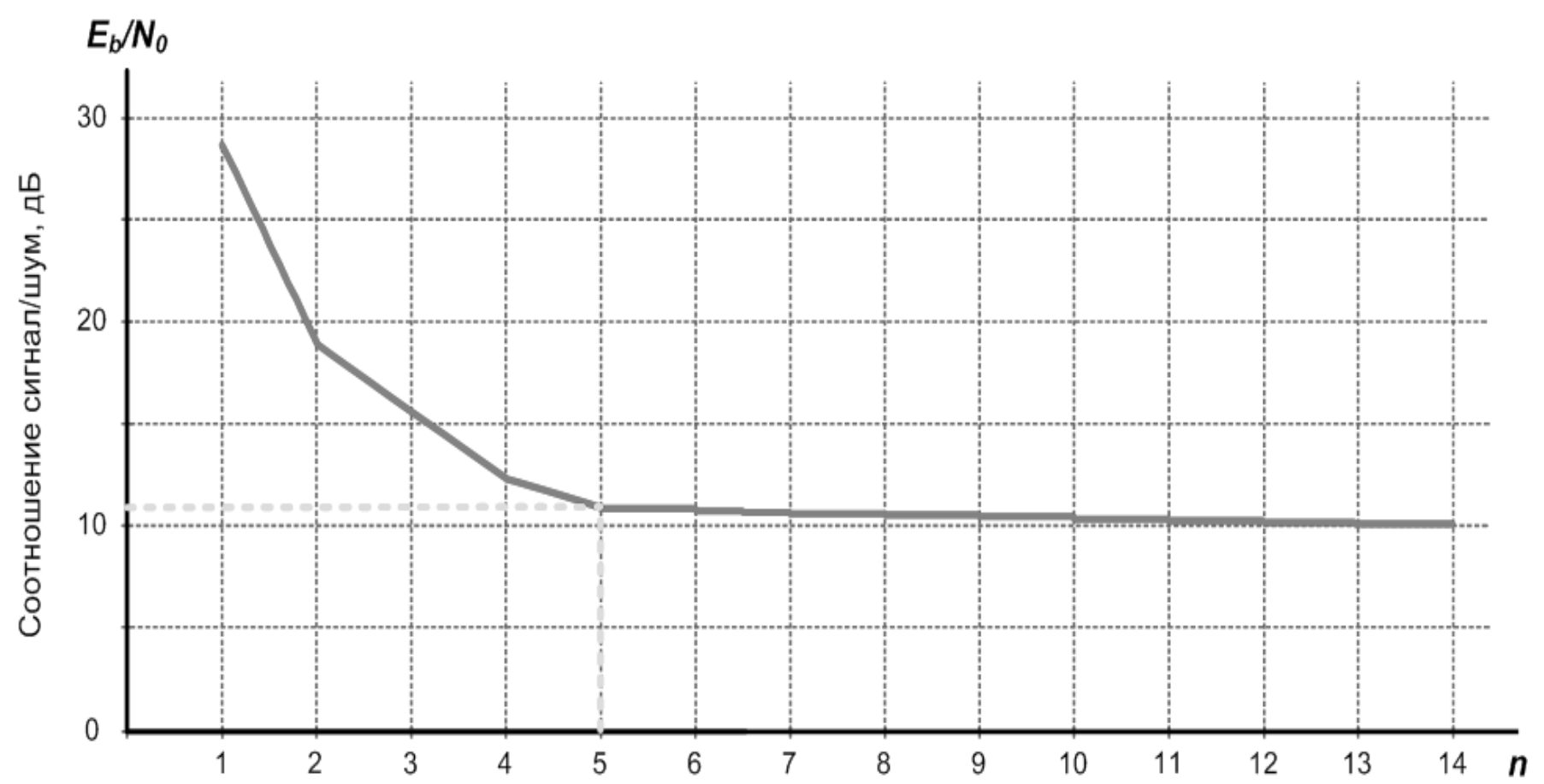

Число антенн на приемной и передающей стороне

Рис. 5. Зависимость числа приёмных и передающих трактов от величины Eb/N0 для систем MIMO, модуляция ДФМ

герентное сложение всех выделенных сигналов $m$ между собой, в результате сложения получается суммарный сигнал тракта $k$, который может быть, в свою очередь, когерентно сложен с сигналами других $n$-1 трактов и итоговый сигнал в блоке 14 на рис. 4 сохраняется в массиве данных $M_{G}$. В блоке 15 на рис. 4 производится демодуляция итогового сигнала из массива данных $M_{G}$ в зависимости от заданного вида модуляции $M$, полученный битовый поток информационных данных будем обозначать $G_{u н \phi}$. При помощи цикла 16 на рис. 4 производится обход всех информационных бит потока $G_{u н ф}$ и в блоке 17 на рис. 4 происходит сравнение их с заданным потоком $R_{\text {инф}}$. Если информационные биты в одинаковых позициях $i$ не равны, то в блоке 18 на рис. 4 производится инкрементирование коэффициента битовой ошибки $K_{Б о}$. Таким образом, по завершению выполнения цикла 16 на рис. 4 и действий внутри него в блоке 19 на рис. 4 переменная коэффициента битовой ошибки $K_{Б о}$ готова для сравнивания с априорно установленным значением $10^{-9}$. В случае, если значение переменной $K_{5 O}$ больше или равно $10^{-9}$, то цель достигнута и данное значение, и значение переменной $n$ записывается в выходной массив данных $\mathrm{M}_{\text {вых } 2}$, блок 20 на рис. 4. В блоке 20 на рис. 4 данные выходного массива $\mathrm{M}_{\text {вых2 }}$, полученные в ходе расчетов, готовы для дальнейшего анализа. По данным выходного массива $\mathrm{M}_{\text {выx } 2}$ на рис. 5 построен график, который наглядно отображает исследуемую зависимость.

Как видно из представленной иллюстрации рис. 5, с увеличением числа передающих и приёмных трактов $n$ системы MIMO, для коэффициента битовой ошибки $\mathrm{K}_{Б O}$ равной $10^{-9}$ значение соотношения $\mathrm{E}_{b} / N_{0}$ уменьшается. По рисунку видно, что при значениях переменной приёмных и передающих трактов, равных более пяти, соотношение $\mathrm{E}_{b} / N_{0}$ уменьшается незначительно.

Заключение. Анализ выходных данных рис. 5, полученных по алгоритму рис. 4 и при помощи разработанной модели радиолинии, показал, что для нужд должностных лиц ситуационных центров комплексной системы безопасности, в частности, для мест, где не проложены ещё оптоволоконные линии связи и для тех мест, где это сделать не представляется возможным в силу известных причин, но требуется использовать современные сервисы связи, автором предлагается использовать разработанную линию радиосвязи с использованием технологии МІМО. Проделанный расчет показал, что наиболее лучшей помехоустойчивостью обладает система MIMO 5×5, поскольку коэффициент битовой ошибки равный $10^{-9}$ был получен для сигнала с соотношением $\mathrm{E}_{b} / N_{0}$, равным 10 дБ. 


\section{ЛИТЕРАТУРА}

1. Викторов В. А. Модель электромагнитных полей, формируемых автоматизированным рабочим местом объекта информатизации // Системы управления, связи и безопасности. 2020. № 1. С. 1-32.

2. Мешалкин В. А. Решение задач электродинамики с помощью вычислительного эксперимента // Журнал научных и прикладных исследований. 2016. Т. 2. № 11 C. 80-82.

3. Ревин С. М. Системно-конфигурационный метод проектирования ситуационных центров в системе комплексной безопасности.- М: Проспект, 2010.147 c.

4. Хореев А. А. Технические каналы утечки акустической (речевой) информации // Специальная техника. 2009. № 5. С. 12-26.

5. Цуканов В. Н. Волоконная оптическая техника.—М: Инфра-Инженерия, 2015.- 243 с.

6. Brown T., Elisabeth C., Persefoni K. Practical Guide to the Mimo Radio Channel with Matlab Examples. — London: John Wiley \& Sons Ltd, 2012 . — 263 p.

7. Shiu D., Foschini G. J., Gans M. J., Kahn J. M. Fading Correlation and Its Effect on the Capacity of Multielement Antenna System // IEEE Transaction On Communications. 2016. Vol. 48. № 2. P. 502-513.

8. Tsoulos G. MIMO System Technology for Wireless Communications. — New York: CRC Press, 2006. - $395 \mathrm{p}$.

9. Zelst A., Hammerschmidt J.S. A single coefficient spatial correlation models for multiple-input multiple output (MIMO) radio channels // Proceeding of the URSI XXVII th General Assembly. (2-5 Aug. 2002). Maastricht, Netherlands, 2002. P. 949-952.

(с) Быданов Егор Владимирович1 ( egorius.90@mail.ru).

Журнал «Современная наука: актуальные проблемы теории и практики»

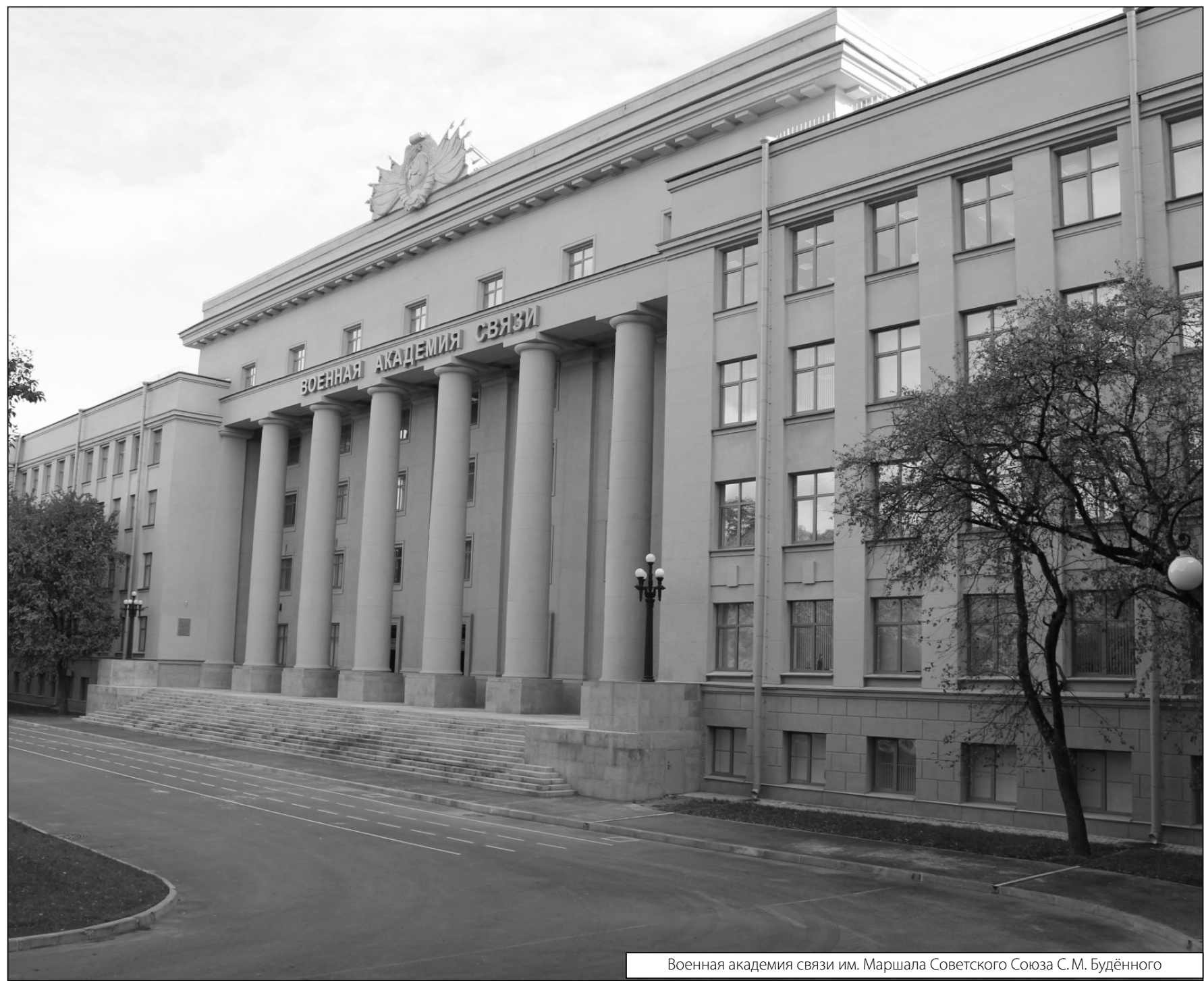

